\section{Addition of phenols compounds to meat dough intended for salami manufacture and its antioxidant effect}

\author{
Enrico Novelli, ${ }^{1}$ Luca Fasolato, ${ }^{1}$ \\ Barbara Cardazzo, ${ }^{1}$ Lisa Carraro, ${ }^{1}$ \\ Agnese Taticchi, ${ }^{2}$ Stefania Balzan'
}

'Dipartimento di Biomedicina Comparata e Alimentazione, Università degli Studi di Padova, Legnaro (PD); ${ }^{2}$ Dipartimento di Scienze Economico-Estimative e degli Alimenti, Università degli Studi di Perugia, Italy

\section{Abstract}

A purified extract of phenols compounds (65\% of phenolic content of which decarboxymethyl oleuropein aglycone represented $45 \%$ of the wet mass) obtained from vegetation water (a by-product of oil mill) was added to a ground meat dough intended for salami manufacture in two concentration levels: 75 and $150 \mathrm{mg} / 100 \mathrm{~g}$ of dough (F1 and F2, respectively). The control batch was composed of lean and fat cuts of pork in 70:30 ratio, $2.7 \%$ salt and a mixed starter culture of staphylococci and pediococci. After stuffing into natural casings, salamis were aged until they reached a total weight loss of $30 \%$. The product was then sliced and packaged in a protective atmosphere (nitrogen:carbon dioxide $80: 20$ ) and placed in a refrigerator thermostat $\left(2-4^{\circ} \mathrm{C}\right)$ with alternating $12 \mathrm{~h}$ of artificial light and darkness. The samples were analysed for the measurement of $\mathrm{pH}$, water activity, organic acidity, peroxide number and secondary products of lipid peroxidation at the time of slicing and after 10, 20 and 30 days of storage into the refrigerated thermostat. The $\mathrm{pH}$ and water activity were not substantially different between the control and the two enriched batches. The peroxide number and secondary products of lipid peroxidation values in the two batches with phenols were at least substantially lower than the control sample. In conclusion, the phenol compounds obtained from vegetation water have shown no interference with the ripening process while protecting the dough from oxidation.

\section{Introduction}

Phenols are synthesised by the plant as a defense against different pathogens. During the early stages of crushing, mixing and extraction of the oil from olive drupe, the bio- transformation of phenolic products of the original fruit occurs (Kubo et al., 1985; Lo Scalzo et al., 1994). Part of these compounds, in view of their strong water solubility will be concentrated in the vegetation water, a mass that is a rejection and as such cannot be released neither into the sewer system nor dispersed in the open field. The vegetation water of the oil mill is a mass containing a solid residue ranging between 6 and just over $7 \%$ and where the phenols represent a percentage between 0.3 and $1.1 \%$ (on wet mass). A suitable multistage filtration process allows to separate most of the solid fraction from the remaining liquid mass of the vegetation water. It is thus possible to lower the organic load of the water to levels consistent with those maximum permitted for the direct entering in the sewerage system. The solid extract which is separated from vegetation water is particularly rich in phenolic compounds, among them decarboxymethyl oleuropein aglycone which represents about $45 \%$ of the mass of the extract in addition to verbascoside, tyrosol and hydroxytyrosol, each one in a residual concentration less than $10 \%$. Phenols compounds have already shown strong antimicrobial and antioxidants properties. The use of enriched extract, such as that from vegetation water, as an ingredient with antioxidant and/or antimicrobial aim in food products may be an example of sustainable use of by-products of the food industry.

\section{Materials and Methods}

\section{Salami manufacture}

A basic formulation consisting of pork lean cuts (shoulder and rump) and fat (belly) in a ratio of about 70:30 was made. The meat was ground using a meat grinder equipped with a plate of $8 \mathrm{~mm}$ holes. The dough (about $30 \mathrm{~kg}$ ) was added of salt (2.7\%) and starter culture (lyophilised mixture of pediococci and staphylococci) and mechanically mixed (batch of control, C). The starter culture added was previously tested in a preliminary evaluation of LAB survival in an environment enriched with phenols compounds. The dough, was divided into three portions of $10 \mathrm{~kg}$ each as follows: $\mathrm{C}=$ control; $\mathrm{F} 1=$ Control+phenol concentration of 75 $\mathrm{mg} / 100 \mathrm{~g}, \mathrm{~F} 2=$ Control+phenol concentration of $150 \mathrm{mg} / 100 \mathrm{~g}$. The stuffing of the dough was carried out using natural casings. The size of the individual units (salami) ranged between 250 and $350 \mathrm{~g}$. The ripening was carried out inside a cabinet equipped with automatic control and regulation of the temperature and relative humidity according to the following program: i) dripping at room temperature for $12 \mathrm{~h}$ without humidity control, ii) drying from 22 to $14^{\circ} \mathrm{C}$ in 8 days and max humidity set at $75 \%$ in the first day and $82 \%$ in the eighth day,
Correspondence: Enrico Novelli, Dipartimento di Biomedicina Comparata e Alimentazione, viale dell'Università 16, 35020 Legnaro (PD), Italy. Tel. +39.049 .8272965 - Fax: +39.0444 .393921$

E-mail enrico.novelli@unipd.it

Key words: Phenols, Vegetation water, Salami, Antioxidant, Lipo-peroxidation.

Conflict of interests: the authors declare no potential conflict of interests.

Received for publication: 12 May 2013 Revision received: 24 December 2013. Accepted for publication: 21 January 2014.

This work is licensed under a Creative Commons Attribution 3.0 License (by-nc 3.0).

(C) Copyright E. Novelli et al., 2014

Licensee PAGEPress, Italy

Italian Journal of Food Safety 2014; 3:1704

doi:10.4081/ijfs.2014.1704

iii) ripening, carried out at a temperature of $14^{\circ} \mathrm{C}$ and maximum relative humidity of $82 \%$, ended on reaching the total weight loss of $30 \%$.

\section{Salami packaging}

An aliquot of each of the three batches of ripened salami was sliced and packaged in a protective atmosphere (a mixture of nitrogen and carbon dioxide in the percentage ratio $80: 20)$ into rigid trays $(176 \times 230 \times 25 \mathrm{~mm})$ closed by hot welding with a sheet of polyethylene plastic film with antifog properties. The trays were placed within a refrigerator thermostat at a temperature of $2-4^{\circ} \mathrm{C}$ and alternating exposure to light (12 $\mathrm{h}$ under light and $12 \mathrm{~h}$ in the dark) obtained by a lamp of $36 \mathrm{~W}, 1600$ lux intended for foods employment. In each tray 9 slices were laid for a total net weight of about $100 \mathrm{~g}$. At the moment of packaging and after 10,20 and 30 days of storage in the refrigerated thermostat a few measurements and analytical determinations were carried out.

\section{Analytical measures}

All slices of each tray (two independent units for each batch) were finely homogenised (2500 rpm 10 s; Retsch, Düsseldorf, Germany). For the measurement of $\mathrm{pH}, 1 \mathrm{~g}$ of the homogenate was then taken immediately, dispersed in water $(1: 10 \mathrm{w} / \mathrm{v})$ and submitted to further crushing using a rod homogeniser $(13,000$ $\mathrm{rpm} / \mathrm{min}$, Ultraturrax T25 Basic; Ika Werke, Staufen, Germany) and the $\mathrm{pH}$ measurement was taken by a Portamess $\mathrm{pH}$-meter (model 910; Knick, Berlin, Germany) coupled with an inLab 427 electrode (Mettler Toledo, Leicester, UK). For the measurement of water activity a teflon capsule was half-filled with homogenised sample and the measure taken using the Aqualab 4 VTE (Decagon Devices, Pullman, WA, USA). The titratable acidity was determi- 
ned on an aliquot of fat (0.5-1 g), previously extracted according to the method of Folch $e t$ al. (1957), solubilised with a mixture of diethyl ether/ethanol (2/1) and titrated with $0.01 \mathrm{M}$ sodium hydroxide in the presence of phenolphthalein. The values have been expressed as percentage of oleic acid. The peroxide number was determined on an aliquot of fat (0.5-1 g) previously extracted from the product and then solubilised with a mixture of acetic acid/chloroform (3/2). After the addition of $0.5 \mathrm{~mL}$ of saturated solution of $\mathrm{KI}$ and incubation in the dark for 2 min, elemental iodine developed by the hydroperoxides that were in the fat sample was titrated by a solution of $0.01 \mathrm{M}$ sodium thiosulphate in the presence of starch as indicator. The results were expressed as meq $\mathrm{O}_{2} / 1000 \mathrm{~g}$ fat. Secondary products of lipid-peroxidation were determined by the thiobarbituric acid reactive substances (TBARs) according to the method of Botsoglou et al. (1994), modified as follows. In a centrifuge tube, $8 \mathrm{~mL}$ of an aqueous solution of $5 \%$ trichloroacetic acid and $5 \mathrm{~mL}$ of $\mathrm{n}$-hexane with $0.008 \%$ Buthylhydroxytoluene (antioxidant agent) were added to approximately $2 \mathrm{~g}$ of the homogenised sample accurately weighed. After homogenisation with Ultraturrax (T25 Basic; Ika Werke) for $30 \mathrm{sec}$ at high speed, the sample was centrifuged for $3 \mathrm{~min}$ at $3000 \mathrm{~g}$ at $4^{\circ} \mathrm{C}$ (Eppendorf 5810R; Eppendorf, Hamburg, Germany) and the surnatant removed. To eliminate the residual solid component, the sample was filtered through paper for rapid filtration. An aliquot of the filtrate $(2.5 \mathrm{~mL})$ was added to $2.5 \mathrm{~mL}$ of a solution of $0.02 \mathrm{M}$ thiobarbituric acid (TBA) and incubated for $35 \mathrm{~min}$ at $95^{\circ} \mathrm{C}$ in a thermostated bath (Julabo ED-13; Seelbach, Baden-Württemberg, Germany). After cooling, the absorbance of the chromatic adduct was read by means of the spectrophotometer Mod. 7800 Jasco UV/VIS (Jasco, Oklahoma City, OK, USA) set at a wavelength of $532 \mathrm{~nm}$. The results were expressed as absorbance normalised to the weight of the sample.

\section{Results}

The $\mathrm{pH}$ values were comprised between 5.21 and 5.27 at the time of the slicing and did not show substantial variations neither among the batches nor as a function of storage time (Table 1). After 30 days, the values ranged between 5.29 and 5.33. Water activity had values between 0.909 and 0.913 for salami just after slicing and there were no marked changes worthy of note after 30 days of storage whose values varied between 0.914 and 0.917 (Table 2). The titratable acidity (expressed as percentage of oleic acid per $100 \mathrm{~g}$ of fat) showed values between 2.95 and $3.18 \%$ at the time of slicing with an increasing trend, as storage time passed, for all the three batches in comparison, reaching final values between 4.59 and $3.88 \%$ at 30 days (Table 3 ). The peroxide number at the time of slicing showed a mean value in the control sample equal to $48.6 \mathrm{meq}$ $\mathrm{O}_{2} / \mathrm{kg}$ fat against lower values in the two batches with phenols added whose values ranged between 12.7 and 19.9 meq $0_{2} / \mathrm{kg}$ fat (Table 4 ). The peroxide number in the control batch

appeared to decrease with the days of storage, whereas it was relatively constant in the samples with phenols added. Finally, the analysis of secondary products of oxidation (i.e. TBARs) showed absorbance values close to 0.1 in the control sample at the beginning, while the batches with added phenols showed values that were $>80 \%$ lower (Table 4 ). This difference was maintained even at the sampling conducted at 10, 20 and 30 days of storage.

Table 1. $\mathrm{pH}$ values of pre-sliced salami packed in protective atmosphere and stored at 2$4^{\circ} \mathrm{C}$ up to 30 days.

\begin{tabular}{lcccc} 
& \multicolumn{4}{c}{ Days of storage } \\
& 0 & 10 & 20 & 30 \\
Control & $5.27 \pm 0.02$ & $5.36 \pm 0.02$ & $5.37 \pm 0.02$ & $5.33 \pm 0.01$ \\
\hline F1 & $5.23 \pm 0.02$ & $5.30 \pm 0.03$ & $5.29 \pm 0.02$ & $5.30 \pm 0.04$ \\
F2 & $5.21 \pm 0.03$ & $5.33 \pm 0.01$ & $5.30 \pm 0.06$ & $5.29 \pm 0.02$ \\
\hline
\end{tabular}

Control, lean and fat cuts of pork in 70:30 ratio, salt 2.7\% and a mixed starter culture of staphylococci and pediococci; F1, $75 \mathrm{mg} / 100 \mathrm{~g}$ of purified extract of phenols compounds added to ground meat dough; $F 2,150 \mathrm{mg} / 100 \mathrm{~g}$ of purified extract of phenols compounds added to ground meat dough. Values are expressed as mean \pm standard deviation.

Table 2. Activity water values of pre-sliced salami packed in protective atmosphere and stored at $2-4^{\circ} \mathrm{C}$ up to 30 days.

\begin{tabular}{lcccc} 
& \multicolumn{4}{c}{ Days of storage } \\
& 0 & 10 & 20 & 30 \\
Control & $0.913 \pm 0.004$ & $0.913 \pm 0.002$ & $0.913 \pm 0.009$ & $0.915 \pm 0.001$ \\
\hline F1 & $0.911 \pm 0.007$ & $0.916 \pm 0.002$ & $0.916 \pm 0.003$ & $0.917 \pm 0.004$ \\
F2 & $0.909 \pm 0.006$ & $0.918 \pm 0.003$ & $0.921 \pm 0.002$ & $0.914 \pm 0.004$ \\
\hline
\end{tabular}

Control, lean and fat cuts of pork in 70:30 ratio, salt $2.7 \%$ and a mixed starter culture of staphylococci and pediococci; $\mathrm{Fl}, 75 \mathrm{mg} / 100 \mathrm{~g}$ of purified extract of phenols compounds added to ground meat dough; F2, $150 \mathrm{mg} / 100 \mathrm{~g}$ of purified extract of phenols compounds added to ground meat dough. Values are expressed as mean \pm standard deviation.

Table 3. Titratable acidity values (\% oleic acid) of pre-sliced salami packed in protective atmosphere and stored at $2-4^{\circ} \mathrm{C}$ up to 30 days.

\begin{tabular}{lcccc} 
& \multicolumn{4}{c}{ Days of storage } \\
Control & 0 & 10 & 20 & 30 \\
\hline F1 & $2.95 \pm 0.40$ & $2.96 \pm 0.44$ & $3.20 \pm 0.07$ & $3.88 \pm 0.11$ \\
F2 & $2.96 \pm 0.22$ & $3.06 \pm 0.60$ & $3.64 \pm 0.17$ & $4.59 \pm 0.14$ \\
\hline
\end{tabular}

Control, lean and fat cuts of pork in 70:30 ratio, salt 2.7\% and a mixed starter culture of staphylococci and pediococci; Fl, $75 \mathrm{mg} / 100 \mathrm{~g}$ of purified extract of phenols compounds added to ground meat dough; F2, $150 \mathrm{mg} / 100 \mathrm{~g}$ of purified extract of phenols compounds added to ground meat dough. Values are expressed as mean \pm standard deviation.

Table 4. Peroxide number (meq $\mathrm{O} 2 / 1000 \mathrm{~g}$ fat) and thiobarbituric acid reactive substances (absorbance value corrected for sample weight) values of pre-sliced salami packed in protective atmosphere and stored at $2-4^{\circ} \mathrm{C}$ up to 30 days.

\begin{tabular}{lcccc} 
& \multicolumn{4}{c}{ Days of storage } \\
& 0 & 10 & 20 & 30 \\
Peroxide number & & & & \\
$\quad$ Control & $48.6 \pm 9.2$ & $33.7 \pm 2.5$ & $12.5 \pm 1.1$ & $19.6 \pm 5.6$ \\
F1 & $12.7 \pm 2.3$ & $8.5 \pm 1.0$ & $15.3 \pm 5.2$ & $12.2 \pm 0.5$ \\
F2 & $19.9 \pm 3.1$ & $6.5 \pm 1.8$ & $18.3 \pm 4.4$ & $15.0 \pm 8.4$ \\
\hline TBARs & & & & \\
Control & $0.090 \pm 0.003$ & $0.134 \pm 0.005$ & $0.135 \pm 0.004$ & $0.157 \pm 0.012$ \\
F1 & $0.017 \pm 0.001$ & $0.014 \pm 0.002$ & $0.020 \pm 0.001$ & $0.026 \pm 0.003$ \\
F2 & $0.014 \pm 0.001$ & $0.015 \pm 0.001$ & $0.013 \pm 0.001$ & $0.023 \pm 0.009$ \\
\hline
\end{tabular}

Control, lean and fat cuts of pork in 70:30 ratio, salt $2.7 \%$ and a mixed starter culture of staphylococci and pediococci; Fl, $75 \mathrm{mg} / 100 \mathrm{~g}$ of purified extract of phenols compounds added to ground meat dough; $F 2,150 \mathrm{mg} / 100 \mathrm{~g}$ of purified extract of phenols compounds added to ground meat dough. Values are expressed as mean \pm standard deviation. 


\section{Discussion}

Apart from what stated in the title and target of the study, a further expected result was the magnitude of the impact played by phenols compounds by a sensory viewpoint. As is apparent from the formulation of the dough, deliberately other ingredients were not been added (for example, pepper, nitrate and sugar) which could in some way interfere with the phenols compounds. In this preliminary evaluation a structured analysis of the sensory characters was not provided. However, a simple evaluation of color, odor and taste, with particular reference to the feeling of bitter and astringent, allowed to identify in the formulation F1 (with the addition of phenols in a concentration of $75 \mathrm{mg} / 100 \mathrm{~g}$ of dough) the best one. The mixture with the double amount of phenols showed, especially to the taste, a bitter aftertaste though not unpleasant in the complex. Differences in terms of smell and taste between F1 and Control were not always recognised by those people who had served the experimental salami for tasting. In certain cases, the dough F1 was scored even higher than the control one in terms of overall satisfaction (data not reported). The values of $\mathrm{pH}$ and water activity were not different between the control and the two batches containing phenols compounds. In other words, there seems to be no interference played by phenols neither respect to the action carried out by starter culture nor to the process of dehydration. Similar results were also observed by Bozkurt (2007) as a result of the addition of sesame oil and Thymbra spicata during the manufacturing of Turkish dry-fermented sausage and the same was observed by Balev et al. (2005) studying the effect of some antioxidants on the lipid and pigment oxidation in dry-fermented sausages. On the other hand, Gök et al. (2011) found an interaction between storage time and antioxidant addition (rosemary and tocopherol) on $\mathrm{pH}$ values of sucuk (Turkish dry-fermented sausage). The lipolytic activity in fermented salami is attributable to the muscular tissue lipase and esterase, a set of enzymes that are very efficient in the early stages of the drying process whereas it becomes prevalent the lipolytic enzymes of the lactic acid bacteria in the later stages of the ripening process. As can be seen for the values at the time zero, the lipolytic activity of the ripened product (measured through titratable acidity) was not compromised in the batches spiked with phenols compounds. However, the lipolytic activity seemed to increase more markedly in the batches enriched with phenols during the following 30 days of storage of the sliced salami packaged in a protective atmosphere. This finding still remains a simple observation with the need to proceed with a deepening of investigation in the course of a subsequent trial. The analysis of peroxides and secondary compounds of lipoperoxidation (Table 4) showed a substantial effect of the phenols added. Similar results were observed by Balev et al. (2005) after the use of rosemary in the amount of $1 \mathrm{~g} / \mathrm{kg}$ of dough for fermented sausages. García-Iñiguez de Ciriano et al. (2009) highlighted a significant reduction in peroxide number through the use of $340 \mathrm{ppm}$ of freeze-dried extract Borago officinalis added to the dough of sausage to be submitted to ripening. Marangoni and de Moura (2011) used essential oil from Coriandrum sativum in Italian salami in the amount of $0.01 \%$ demonstrating the effectiveness of this natural antioxidant against the oxidation of the lipid fraction of the meat mixture. The results reported in Table 4 , for the number of peroxides, also show that the stabilising effect of the lipid by phenolic compounds was much more evident when the oxidative attack occurs by radical action, as is the case of the stuffed product (as can be seen by the values at time zero when the salami were sliced just at the end of ripening). Instead, in the case of the salami that have been sliced, packaged and then stored under refrigeration and exposed to light for at least $12 \mathrm{~h} /$ day, the photooxidation pathway seemed to be less affected by the protective action of phenolic compounds. Although there is no statistical support, the difference between the control batch and those with phenols at time zero (autoxidation via radical attack) was much greater than the difference observed for the packaged salami after 30 days of storage (photo-oxidation pathway).

The secondary compounds of lipo-peroxidation reflected the situation already observed for the peroxides number. Both the minimum load of peroxides at the end of ripening in the batches with phenol compounds and a minimum, but still significant, reduction of the lipo-peroxidation during the refrigerated storage of sliced product ensured a clear slowdown in the process of rancidity. The phenols added to the dough therefore appeared to exert an excellent antioxidant effect. Also significant was the substantial similarity in the results between the two experimental batches (F1 and F2), which corroborates the hypothesis of an effective antioxidant effect at phenols concentration compatible with the maintenance of a more than acceptable level of quality in terms of sensory tasting.

\section{Conclusions}

The phenolic compounds obtained by the concentration and purification of the solid fraction of the vegetation water of the oil mill have shown a clear and effective antioxidant capacity in a preliminary evaluation carried out directly in the food matrix. Equally important was found to be also their compatibility with the lactic acid bacteria which are due the processing and shelf-life of the salami.

\section{References}

Balev D, Vulkova T, Dragoev S, Zlatanov M, Bahtchevanska S, 2005. A comparative study on the effect of some antioxidants on the lipid and pigment oxidation in dryfermented sausages. Int J Food Sci Tech 40:977-83.

Botsoglou NA, Fletouris DJ, Papageorgiou GE, Vassilopoulos VN, Mantis AJ, Trakatellis AG, 1994. Rapid, sensitive, and specific thiobarbituric acid method for measuring lipid-peroxidation in animal tissue, food, and feedstuff samples. J Agr Food Chem 42:1931-7.

Bozkurt H, 2007. Comparison of the effects of sesame and Thymbra spicata oil during the manufacturing of Turkish dry-fermented sausage. Food Control 18:149-56.

Folch J, Less M, Stanley GHS, 1957. A simple method for the isolation and purification of total lipids from animal tissues. J Biol Chem 226:497-509.

García-Iñiguez de Ciriano M, García-Herreros C, Larequi E, Valencia I, Ansorena D, Astiasarán I, 2009. Use of natural antioxidants from lyophilized water extracts of Borago officinalis in dry fermented sausages enriched in w-3 PUFA. Meat Sci 83:271-7.

Gök V, Obuz E, Ekin Şahin M, Serteser A, 2011. The effects of some natural antioxidants on the color, chemical and microbiological properties of sucuk (Turkish dry-fermented sausage) during ripening and storage periods. J Food Process Pres 35:677-90.

Kubo I, Matsumoto A, Takase I, 1985. A multichemical defense mechanism of bitter olive Olea europaea (Oleaceae). Is oleuropein a phytoalexin precursor? J Chem Ecol 11:251-62.

Lo Scalzo R, Scarpati ML, Verzegnassi B, Vita G, 1994. Olea europaea chemicals repellent to Dacus oleae females. J Chem Ecol 20:1813-23.

Marangoni C, de Moura NF, 2011. Antioxidant activity of essential oil from Coriandrum Sativum L. in Italian salami. Cienc Tecnol Aliment 31:124-8. 